\title{
Cross-like terahertz metamaterial absorber for sensing applications
}

\author{
CUMALI SABAH ${ }^{1,2} \mathbb{E}$,*, BATUHAN MULLA ${ }^{3}$, HAKAN ALTAN $^{4}$ and LUTFI OZYUZER 5 \\ ${ }^{1}$ Department of Electrical and Electronics Engineering, Middle East Technical University-Northern Cyprus Campus \\ (METU-NCC), Kalkanlı, Guzelyurt, TRNC/Mersin 10, Turkey \\ ${ }^{2}$ Kalkanli Technology Valley (KALTEV), Middle East Technical University-Northern Cyprus Campus (METU- \\ NCC), Kalkanlı, Guzelyurt, TRNC/Mersin 10, Turkey \\ ${ }^{3}$ Sustainable Environment and Energy Systems, Middle East Technical University-Northern Cyprus Campus \\ (METU-NCC), Kalkanlı, Guzelyurt, TRNC/Mersin 10, Turkey \\ ${ }^{4}$ Department of Physics, Middle East Technical University, 06800 Cankaya, Ankara, Turkey \\ ${ }^{5}$ Department of Physics, Izmir Institute of Technology, 35430 Urla, Izmir, Turkey \\ *Corresponding author. E-mail: sabah@metu.edu.tr
}

MS received 25 May 2017; revised 13 December 2017; accepted 12 January 2018; published online 20 June 2018

\begin{abstract}
In this work, a new multiband terahertz metamaterial absorber is designed and characterised by numerical simulation method. In addition, the utilisation of the proposed absorber as a sensor is also investigated. The dielectric and thickness sensing characteristics are analysed. The proposed multiband metamaterial absorber has the ability for utilising the terahertz region up to $2 \mathrm{THz}$. According to the results, it is found that the proposed absorber is capable of sensing unknown materials and material thickness with any of its five absorption bands. The sensitivity of the proposed sensor is $6.57 \mathrm{GHz} /$ unit sensitivity for dielectric sensing and $7.66 \mathrm{GHz} / \mu \mathrm{m}$ for thickness sensing.
\end{abstract}

Keywords. Metamaterial; absorber; sensor; multiband; terahertz waves.

PACS Nos 78.67.Pt; 07.07.Df; 78.20.Bh; 07.05.Tp

\section{Introduction}

Metamaterials which are artificially designed materials and have the geometric scales smaller than the wavelength of incoming electromagnetic waves, can show some exotic and naturally unavailable properties like double negativity $[1,2]$, negative refraction $[3,4]$, invisibility cloaking [5] etc. Due to these properties, there are many studies in the literature for different frequency ranges like gigahertz $[6,7]$, optical $[8,9]$ and terahertz $[10,11]$.

The designs engineered for utilising the terahertz region of the electromagnetic spectrum (the so-called terahertz gap) have attracted a great deal of interest [10-12]. This is mainly because of the potential application in imaging, sensing and absorbing property in this region. Metamaterials can be used to utilise terahertz gap region.

Absorbing property of the metamaterials will be perfect, when the effective impedance of the designed absorber structure is the same as the impedance of the empty space $[13,14]$. Under this condition, the transmitted and the reflected electromagnetic radiations minimise simultaneously.

The geometry of the metamaterial absorbers can be modified so that a specific electromagnetic response can be obtained at a certain frequency. These absorbers can be used for sensing, imaging, absorber applications with different characteristics like polarisation independent, multiband or wideband [15-18].

Metamaterial absorbers can be good candidates in terahertz region for chemical and biological sensing [19-21]. When an unknown material or analyte is added, metamaterials can analyse and investigate the unknown material by giving response in the resonance frequency. Because of the added unknown material, interaction occurs between this unknown material and the metamaterial absorber. As a result of this interaction, frequency shifts occur. By analysing these shifts, the sensing property can be realised [22].

In this work, a new multiband metamaterial absorber for terahertz regime is designed and characterised by 
numerical simulation method. In addition, the utilisation of the proposed absorber as a sensor for both dielectric and thickness sensing are also investigated.

\section{Design and simulation}

The perspective and the front views (as well as the geometric parameters) of the unit cell configurations of the metamaterial absorber are presented in figures $1 \mathrm{a}$ and $1 \mathrm{~b}$ respectively. The geometric design of the structure consists of a gold $(\mathrm{Au})$ plate at the bottom, a quartz dielectric spacer layer, and a gold cross resonator at the top. The unique gold resonator arise from the combination of two cross strips and semicircular gold patches at the edges of each of the cross strips. The geometrical parameters of this design are demonstrated in figure $1 \mathrm{~b}$. Here, ' $\mathrm{x}$ ' represents the unit cell periodicity, ' $r$ ' is the dimeter of the semicircular patches, ' $t$ ' indicates the width of the strips, ' 1 ' represents the length of the strips. The values associated with these parameters are: $x=300 \mu \mathrm{m}$, $r=30 \mu \mathrm{m}, l=185 \mu \mathrm{m}, t=12 \mu \mathrm{m}$. In addition to these parameters, the thickness of the metallic parts are $1 \mu \mathrm{m}$ and the thickness of the quartz layer is $20 \mu \mathrm{m}$.

The numerical characterisation of the metamaterial absorber is performed by an electromagnetic simulation software based on finite integration method. Gold with the conductivity of $4.09 \times 10^{7} \mathrm{~S} / \mathrm{m}$, quartz with the dielectric constant of 3.75 and loss tangent of 0.004 are used to introduce the mentioned materials in the simulation software [23-25]. For the simulations, periodic boundaries are used in $x$ and $y$ directions and in $z$ directions open boundaries are employed in order to ensure the periodically simulated design. The polarisation of the electromagnetic field is given in figure 1. Through all the simulations the wave is a transverse electromagnetic wave.

In general, the absorption characteristics which rely on frequency can be obtained from eq. (1).

(a)

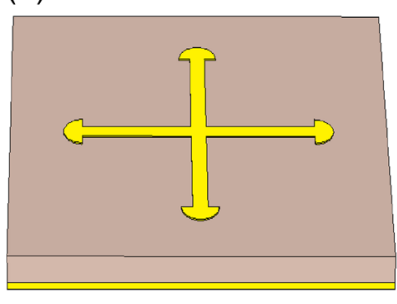

Transmission + Reflection + Absorption $=1$.

In this relation, absorption, reflection and transmission are represented by $A(\omega), R(\omega), T(\omega)$ respectively. It is obvious from this equation that if one wants to maximise $A(\omega), R(\omega)=\left|S_{11}\right|^{2}$ and $T(\omega)=\left|S_{21}\right|^{2}$ should be minimised simultaneously. As the bottom metallic plate of the proposed metamaterial absorber is sufficiently thick, it behaves like an optical mirror. Therefore, this condition ensures the elimination of transmissions from eq. (1). Hence, the scattering parameters $\left(S_{11}\right)$ obtained from the numerical analysis will be adequate to calculate the optical absorption response of the design in the subterahertz regime.

\section{Results and discussion}

According to the simulation results, the proposed metamaterial absorber shows nine absorption peaks between 0 and $2 \mathrm{THz}$ as presented in figure 2 . However, only five of these absorption peaks have absorption rates greater than $90 \%$. These resonances take place at $0.370 \mathrm{THz}$, $1.062 \mathrm{THz}, 1.362 \mathrm{THz}, 1.670 \mathrm{THz}$ and $1.776 \mathrm{THz}$ with $94.16 \%, 96.01 \%, 97.99 \%, 96.51 \%$ and $99.68 \%$ absorption levels. For simplicity, these five peaks will be used in the analysis.

In order to show better understanding of the absorption behaviour of the design, field distributions at resonant frequencies are obtained and presented in figure 3. Figures $3 a_{1}-3 a_{5}$ give the electric field distributions at $f=0.37 \mathrm{THz}, 1.06 \mathrm{THz}, 1.36 \mathrm{THz}, 1.67 \mathrm{THz}$ and $1.78 \mathrm{THz}$, respectively, while figures $3 \mathrm{~b}_{1}-3 \mathrm{~b}_{5}$ monitor the magnetic field distributions at $f=0.37 \mathrm{THz}$, $1.06 \mathrm{THz}, 1.36 \mathrm{THz}, 1.67 \mathrm{THz}$ and $1.78 \mathrm{THz}$, respectively.

For the first resonance at $0.37 \mathrm{THz}$, the electric field is mainly concentrated on and near the horizontal strip as given in figure $3 a_{1}$. The accumulation of the electric

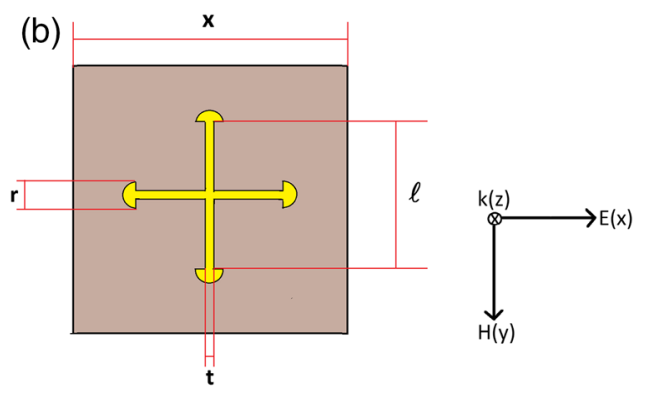

Figure 1. Geometry of the unit cell of the proposed metamaterial absorber. (a) Perspective view and (b) front view ( $x=300 \mu \mathrm{m}, r=30 \mu \mathrm{m}, t=12 \mu \mathrm{m}, l=185 \mu \mathrm{m}$, thickness of the metallic parts: $1 \mu \mathrm{m}$, thickness of quartz: $20 \mu \mathrm{m})$. Incident wave polarisation is given on the right. 


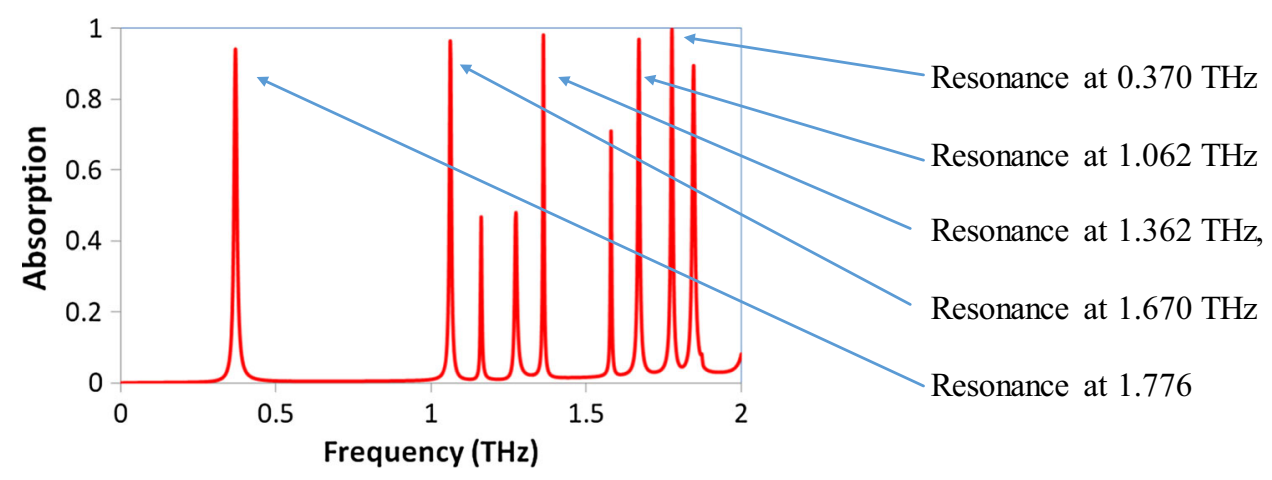

Figure 2. Absorption spectrum of the proposed absorber.
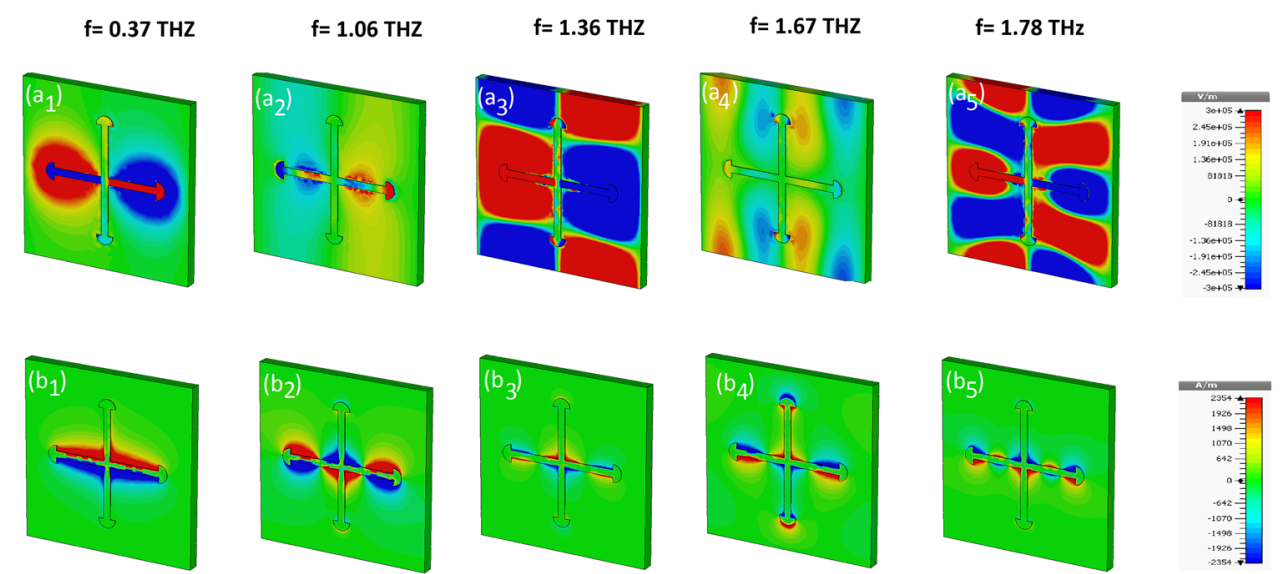

Figure 3. Z-component of the field distributions at resonant frequencies. $\left(\mathbf{a}_{1}-\mathbf{a}_{5}\right)$ Electric field distributions at $f=0.37 \mathrm{THz}$, $1.06 \mathrm{THz}, 1.36 \mathrm{THz}, 1.67 \mathrm{THz}$ and $1.78 \mathrm{THz}$ respectively and $\left(\mathbf{b}_{\mathbf{1}}-\mathbf{b}_{\mathbf{5}}\right)$ magnetic field distributions at $f=0.37 \mathrm{THz}, 1.06 \mathrm{THz}$, $1.36 \mathrm{THz}, 1.67 \mathrm{THz}$ and $1.78 \mathrm{THz}$, respectively.

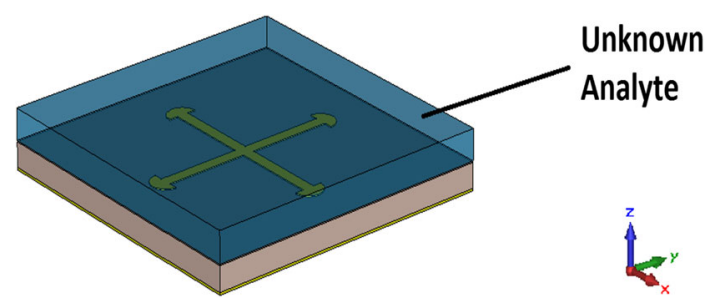

Figure 4. Illustration of over-layer for sensing applications.

field in this way, results in the separation of opposite charges so that the design behaves as an electric dipole. Furthermore, another electric dipole is also generated around the edges of the horizontal strip due to the concentration of electric field at the opposite sides. In figure $3 b_{1}$, the magnetic field is mainly confined on the bottom and the top sides of the horizontal strip, forming a magnetic dipole. When the electric and magnetic responses are combined, this condition provides perfect absorption due to the impedance matching. Note that the impedance of the design rely on the electric and magnetic responses $(z(w)=\sqrt{ }((\mu(w)) /(\varepsilon(w))))$. Hence,

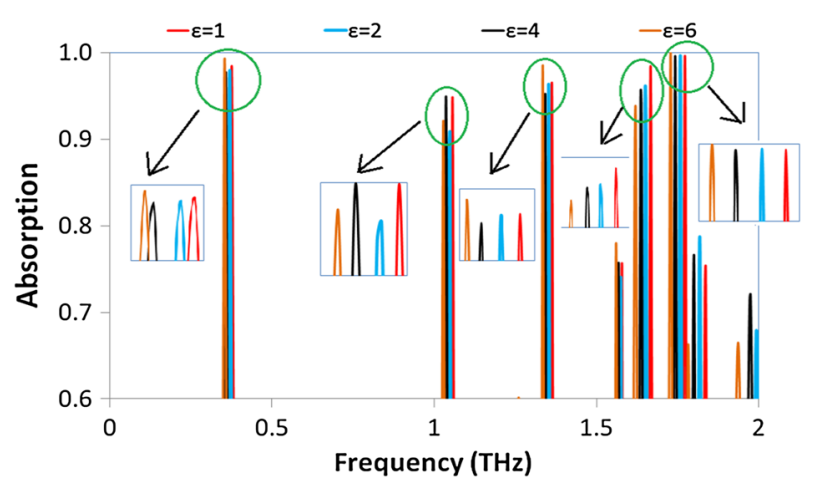

Figure 5. Variation of absorption spectra when different dielectric constants are used. The inserted plots represent the zoom of the circled areas.

impedance matching condition can be fulfilled when the electric and magnetic responses exist at the same time. Thus, under these circumstances, zero reflection and transmission of the incident wave can be obtained. Note that, in order to fulfil the matching condition, the normalised impedance should be purely real $(\operatorname{Re}(z) \approx 1$ and $\operatorname{Im}(z) \approx 0)$ [26]. For the second resonance at 
(a)

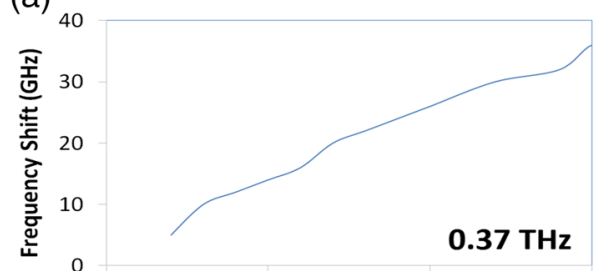

(c)

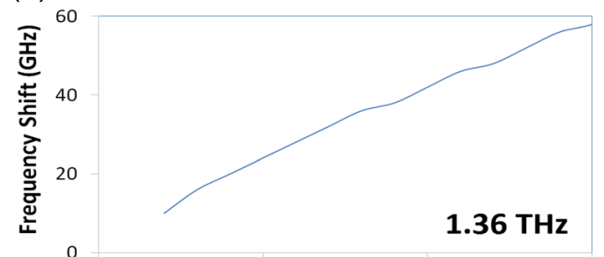

(e)

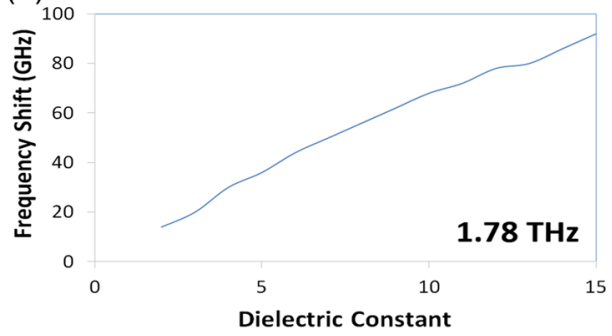

(b)



(d)

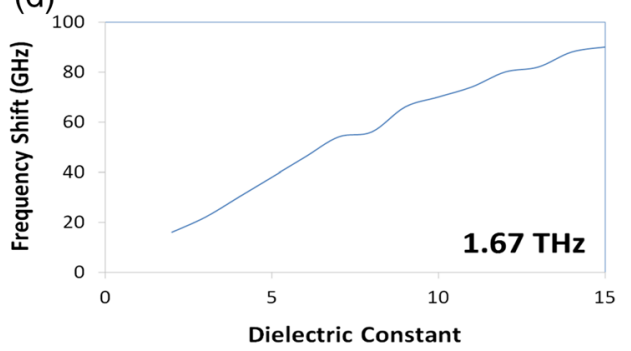

Figure 6. Frequency shift as a function of the dielectric constant of the over-layer for the resonance at (a) $0.37 \mathrm{THz},(\mathbf{b})$ $1.06 \mathrm{THz},(\mathbf{c}) 1.36 \mathrm{THz},(\mathbf{d}) 1.67 \mathrm{THz}$ and $(\mathbf{e}) 1.78 \mathrm{THz}$.

$1.06 \mathrm{THz}$, the electric field distribution (figure $3 \mathrm{a}_{2}$ ) suggests two dipole resonances; the first one occurs between the two horizontal semicircular patches and the second one can be observed on the portions of the horizontal strips between the mid-points of the vertical strips and the semicircular patches. However, when the magnetic field in figure $3 b_{2}$ is analysed, it can be seen that the magnetic field shows hexapolar characteristics. Therefore, for the second resonance it can be commented that excitation of the electric and magnetic resonances simultaneously give rise to an absorption resonance at $1.06 \mathrm{THz}$. For the resonance at $1.36 \mathrm{THz}$, the half-wave resonance modes can be seen at the upper and lower portions of the design for electric field (figure $3 a_{3}$ ). When figures $3 a_{3}$ and $3 a_{5}$ are compared, it can be seen that figure $3 \mathrm{a}_{5}$ represents the higher-order mode of figure $3 a_{3}$. In fact, higherorder modes arise at higher frequencies [27-31]. In addition to the electric field, magnetic responses (figures $3 b_{3}$ and $3 b_{5}$ ) show similar behaviour. Therefore, these two resonances exhibit the same characteristics. However, for the resonance at $1.67 \mathrm{THz}$, the electric response is mostly generated by the vertical semicircular patches and the edges of the design (figure $3 \mathrm{a}_{4}$ ) which indicates the presence of octopolar electric field distributions. For the fourth resonance, not only the electric field but also the magnetic field gives octopolar responses.

\subsection{Sensor applications}

For sensor application, an unknown layer is added on the top of the proposed metamaterial absorber and simulations are carried out according to this. The illustration of the over-layer is presented in figure 4 . In order to reveal the sensing characteristics of the absorber, the permittivity of the unknown layer is altered and the variation of the absorption spectrum is investigated. In addition to the permittivity, the thickness of the over-layer is also altered and the variation of the absorption characteristics are examined. Moreover, the sensitivity of the proposed metamaterial absorber sensor is calculated for five absorption resonances.

For permittivity sensing, the dielectric constant of the over-layer is altered between 1 and 15 with the step of 1. The loss tangent has been taken as fixed at 0.02 in each of the cases. The thickness of the unknown layer is assumed to be $1 \mu \mathrm{m}$. The absorption results corresponding to the variation of the dielectric constants are given in figure 5. For simplicity, some of the absorption results are presented in this figure. When the dielectric constant is increased, it is observed that the resonances are shifting to the left side (redshift) of the spectrum. This is because of the change in the total capacitance of the structure. It is also known that the resonant frequencies are strongly dependent on the total capacitance of the structure. When the structure is coated with an unknown 
Table 1. Normalised sensitivity of the proposed sensor at various resonance frequencies, when the dielectric permittivity is altered between 1 and 15 .

\begin{tabular}{llllll}
\hline Resonance frequency (THz) & 0.370 & 1.062 & 1.362 & 1.670 & 1.776 \\
Normalised sensitivity (GHz/PU) & 6.940 & 4.440 & 3.040 & 3.860 & 3.700 \\
\hline
\end{tabular}

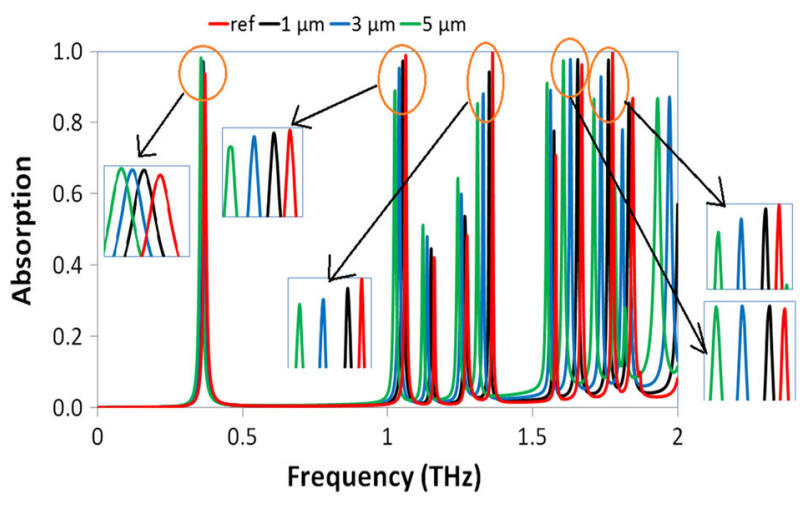

Figure 7. Variation of absorption spectra when over-layers with different thicknesses are used. The inserted plots represent the zoom of the circled areas.

layer, the capacitance of the design increases and therefore, the resonance frequencies shift downwards [22].

In addition, the frequency shifts as a function of the dielectric constant of over-layer for all five resonances are presented in figure 6 . In general, all the five resonances exhibit linear behaviour when the dielectric constants are between 0 and 15 . In order to make distinctions between the sensitivities of the resonances, normalised sensitivities for the five resonances have been calculated and presented in table 1 .

According to the normalised sensitivity data presented in table 1 , the highest sensitivity is observed at $0.370 \mathrm{THz}$ with the magnitude of $6.94 \mathrm{GHz} /$ permittivity unit (PU). Therefore, it can be stated that the use of the first resonance will ensure more accurate results.

For thickness sensing, the analysis is carried out for two different thickness regions; when the thickness is lower than $5 \mu \mathrm{m}$ and when the thickness is lower than $10 \mu \mathrm{m}$. In both cases, the dielectric constant of the overlayer material is assumed to be 2 with a loss tangent of 0.02 . Some of the results for the absorption spectra when various thicknesses of over-layer were used are given in figure 7 . The inserted plots in figure 7 show the zoom of the circled areas for better sight. Similar to the results obtained in figure 5, the absorption spectra show redshift when the thicknesses of over-layer are increased. This condition can also be explained by the capacitance increment. The little fluctuations in the absorption rates are due to the better or worse excitation of the electric and magnetic responses. For five absorption peaks, the thickness sensitivity values are calculated and given in table 2 for the mentioned cases.

Frequency shifts exhibit different characteristics under different over-layer thicknesses. Therefore, normalised sensitivity data are calculated and analysed for two different layer thicknesess. Calculated sensitivity results suggest up to $7.66 \mathrm{GHz} / \mu \mathrm{m}$ sensitivity at $1.67 \mathrm{THz}$, when an over-layer thinner than $5 \mu \mathrm{m}$ was used. However, the second absorption resonance at $1.362 \mathrm{THz}$ provides $7.64 \mathrm{GHz} / \mu \mathrm{m}$ which is very close to the highest sensitivity result. Therefore, frequency shift as a function of the thickness of the over-layer are given for only these two resonances. Figure $8 \mathrm{a}$ is given for $1.6362 \mathrm{THz}$ while figure $8 \mathrm{~b}$ is for $1.67 \mathrm{THz}$. Moreover, the highest sensitivity becomes $7.64 \mathrm{GHz} / \mu \mathrm{m}$ at $1.362 \mathrm{THz}$ when an over-layer thinner than $10 \mu \mathrm{m}$ was used. As a result, the sensing of unknown material in the terahertz frequency region with desired coverage thicknesses can be obtained. In addition to the frequency shift, the resonance frequencies as a function of over-layer thicknesses are also presented within these plots. It can be observed from figure 8 that, the frequency shift as a function of thickness has a similar linear behaviour for all the three plots. However, in figure 8a, the shift in the resonant frequency is not much but for other resonances the magnitude of the absorption peaks become smaller as the thickness increases.

As a result, when all the obtained results are combined for sensitivity, the first resonance $(0.37 \mathrm{THz})$ provides the best values for dielectric sensing with the sensitivity of $6.94 \mathrm{GHz} / \mathrm{PU}$ while the resonance at $1.362 \mathrm{THz}$ provides the best sensitivity values for thickness sensing. It ensures higher values for both thicknesses with the sensitivity of $7.64 \mathrm{GHz} / \mu \mathrm{m}$ sensitivity. In addition to these, figure of merit (FoM) was calculated for thickness sensing. It is found that the highest FoM is obtained for the third resonance at $1.337 \mathrm{THz}$ with the FoM value of 1.50 .

\section{Conclusion}

In conclusion, in this paper the numerical simulation of a metamaterial absorber for absorber and sensing application are carried out. By utilising the designed structure, sensing in the terahertz region can be realised. In this paper, the sensing property was analysed in two different 
Table 2. Normalised sensitivity values of the proposed sensor at various resonance frequencies when the thickness of the over-layer is $<5 \mu \mathrm{m}$ and $<10 \mu \mathrm{m}$.

\begin{tabular}{lccccc}
\hline & \multicolumn{5}{c}{ Sensitivity $(\mathrm{GHz} / \mu \mathrm{m})$} \\
\cline { 2 - 6 } Thickness $(\mu \mathrm{m})$ & $0.37 \mathrm{THz}$ & $1.062 \mathrm{THz}$ & $1.362 \mathrm{THz}$ & $1.67 \mathrm{THz}$ & $1.776 \mathrm{THz}$ \\
\hline Normalised $<5$ & 7.57 & 6.40 & 7.64 & 7.66 & 7.21 \\
Normalised $<10$ & 4.86 & 5.84 & 7.64 & 6.11 & 6.87 \\
\hline
\end{tabular}

(a)

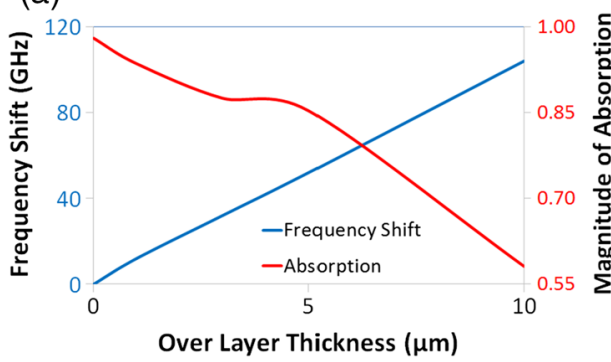

(b)

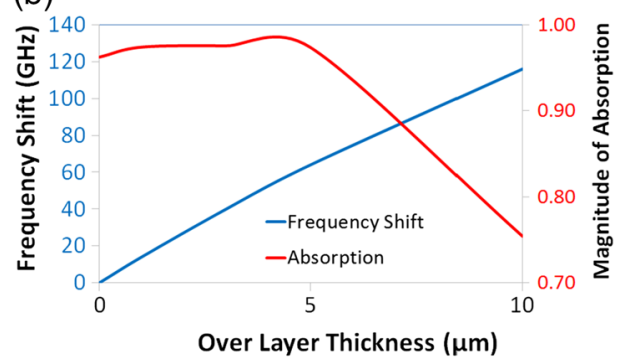

Figure 8. Frequency shift and the variation of resonance frequency as a function of over-layer thickness for the resonance at (a) $1.362 \mathrm{THz}$ and (b) $1.67 \mathrm{THz}$.

conditions for different resonant frequencies; dielectric sensing and thickness sensing. The sensitivity results indicate that the best sensing can be obtained when the resonance at $0.37 \mathrm{THz}$ is used for dielectric sensing. In addition to this, if the thickness of the unknown material is lower than $5 \mu \mathrm{m}$, the third and the fourth resonances can be employed to obtain higher rates of sensitivity. However, when the thickness of the unknown material reaches up to $10 \mu \mathrm{m}$, third resonance can be employed. In addition to the sensing application, the proposed metamaterial absorber can also be used for absorber application.

\section{Acknowledgements}

The study reported here was carried out at Middle East Technical University-Northern Cyprus Campus (METU-NCC). It is supported by METU-NCC under the grant numbers of BAP-FEN-15-D-3 and BAPFEN-16-K-8; and partially supported by TUBITAK (Scientific and Technical Research Council of Turkey) under the project number of 114F091.

\section{References}

[1] R W Ziolkowski, IEEE T. Antenn. Propag. 5, 1516 (2003)

[2] N Engheta and R W Ziolkowski, IEEE T. Microw. Theory $\mathbf{5 3}, 1535$ (2005)
[3] J Valentine, S Zhang, T Zentgraf, E Ulin-Avila, D A Genov, G Bartal and X Zhang, Nature 455, 376 (2008), https://doi.org/10.1038/nature07247

[4] C M Watts, X Liu and W J Padilla, Adv. Mater. 24, OP98 (2012)

[5] D Schurig, J J Mock, B J Justice, S A Cummer, J B Pendry, A F Starr and D R Smith, Science 314, 977 (2006)

[6] W Li, X Zhou, Y Ying, X Qiao, F Qin, Q Li and S Che, AIP Adv. 5, 067151 (2015)

[7] F Dincer, M Karaaslan, E Unal, K Delihacioglu and C Sabah, Prog. Electromagn. Res. 144, 123 (2014)

[8] B Mulla and C Sabah, Waves Random. Complex. 25, 382 (2015), https://doi.org/10.1080/17455030.2015. 1042091

[9] B Mulla and C Sabah, Plasmonics 11, 1313 (2016), https://doi.org/10.1007/s11468-015-0177-y

[10] X He, S Yan, Q Ma, Q Zhang, P Jia, F Wu and J Jiang, Opt. Commun. 340, 44 (2015)

[11] B Wang, X Zhai, G Wang, W Huang and L Wang, Opt. Mater. Express 5, 227 (2015)

[12] Wei Su and Bingyan Chen, Pramana - J. Phys. 89, 37 (2017), https://doi.org/10.1007/s12043-017-1436-6

[13] X Liu, T Tyler, T Starr, A F Starr, N M Jokerst and W J Padilla, Phys. Rev. Lett. 107, 045901-1-4 (2011)

[14] S Gu, J P Barrett, T H Hand, B-I Popa and S A Cummer, J. Appl. Phys. 108, 064913-1-6 (2010)

[15] A Díaz-Rubio, A P Hibbins, J Carbonell and J SánchezDehesa, Appl. Phys. Lett. 106, 241604 (2015)

[16] J B Pendry, D Schurig and D R Smith, Science 312, 1780 (2006)

[17] W Cai, D A Genov and V M Shalaev, Phys. Rev. B 72, 193101 (2005) 
[18] N I Landy, S Sajuyigbe, J J Mock, D R Smith and W J Padilla, Phys. Rev. Lett. 100, 207402 (2008)

[19] Xunjun He, Qinfei Zhang, Guangjun Lu, Guobing Ying, Fengmin $\mathrm{Wu}$ and Jiuxing Jiang, RSC Adv. 6, 52212 (2016)

[20] C Sabah and H G Roskos, Microsyst. Technol. 18, 2071 (2012)

[21] L Cong, Siyu Tan, Riad Yahiaoui, Fengping Yan, Weili Zhang and Ranjan Singh, Appl. Phys. Lett. 106, 031107 (2015)

[22] Cumali Sabah and Hartmut G Roskos, Eur. Phys. J. Appl. Phys. 61, 30402 (2013)

[23] Ben-Xin Wang, Ling-Ling Wang, Gui-Zhen Wang, WeiQing Huang, Xiao-Fei Li and Xiang Zhai, IEEE Photon. Technol. Lett. 26(2), 111 (2014)

[24] Ben-Xin Wang, Gui-Zhen Wang and Ling-Ling Wang, Plasmonics 11, 523 (2016), https://doi.org/10.1007/ s11468-015-0076-2
[25] Hu Tao, C M Bingham, D Pilon, Kebin Fan, A C Strikwerda, D Shrekenhamer, W J Padilla, Xin Zhang and R D Averitt, J. Phys. D: Appl. Phys. 43, 225102 (2010)

[26] Y Ra'di, C R Simovski and S A Tretyakov, Phys. Rev. Appl. 3, 037001 (2015)

[27] B X Wang, X Zhai, G Z Wang, W Q Huang and $\mathrm{L} \mathrm{L}$ Wang, IEEE Photon. J. 115, 1 (2015)

[28] D Hu, H Y Wang and Q F Zhu, IEEE Photon. J. 8, 5500608 (2016)

[29] G Dayal and S A Ramakrishna, J. Opt. 16, 094016 (2014)

[30] B X Wang, IEEE J. Sel. Top. Quantum Electron. 23, 4700107 (2017)

[31] Yong Zhi Cheng, Mu Lin Huang, Hao Ran Chen, Zhen Zhong Guo, Xue Song Mao and Rong Zhou Gong, https://doi.org/10.20944/preprints201703.0022.v1 